\title{
BMJ Open Quality Implementation of a rapid response system at an isolated radiotherapy facility through simulation training
}

\author{
Hidemasa Kawamura (10 , ${ }^{1}$ Yasuhiro Komatsu, ${ }^{2}$ Kazumi Tanaka, ${ }^{2}$ \\ Masafumi Kanamoto, ${ }^{3}$ Masaru Tobe, ${ }^{3}$ Chisato Usami, ${ }^{3}$ Hiroshi Hinohara, ${ }^{3}$ \\ Kiyohiro Oshima, ${ }^{4}$ Yoko Kitada, ${ }^{1}$ Kazuhisa Tsuda, ${ }^{1}$ Tomoaki Ogano, ${ }^{1}$ \\ Hirofumi Shimada, ${ }^{1}$ Tatsuya Ohno ${ }^{1}$
}

\begin{abstract}
To cite: Kawamura $\mathrm{H}$, Komatsu Y, Tanaka K, et al. Implementation of a rapid response system at an isolated radiotherapy facility through simulation training. BMJ Open Quality 2022;11:e001578. doi:10.1136/ bmjoq-2021-001578
\end{abstract}

Received 3 June 2021 Accepted 21 December 2021

Check for updates

(C) Author(s) (or their employer(s)) 2022. Re-use permitted under CC BY-NC. No commercial re-use. See rights and permissions. Published by BMJ.

${ }^{1}$ Gunma University Heavy lon Medical Center, Maebashi, Japan

${ }^{2}$ Department of Healthcare Quality and Safety, Gunma University Graduate School of Medicine, Maebashi, Japan ${ }^{3}$ Intensive Care Unit, Gunma University Hospital, Meabashi, Japan

${ }^{4}$ Emergency Medical Center, Gunma University Hospital, Meabashi, Japan

Correspondence to Dr Hidemasa Kawamura; kawa@gunma-u.ac.jp

\section{ABSTRACT}

A rapid response system is required in a radiotherapy department for patients experiencing a critical event when access to an emergency department is poor due to geographic location and the patient is immobilised with a fixation device. We, therefore, rebuilt the response system and tested it through onsite simulations. A multidisciplinary core group was created and onsite simulations were conducted using a Plan-Do-Study-Act cycle. We identified the important characteristics of our facility, including its distance from the emergency department; the presence of many staff with little direct contact with patients; the treatment room environment and patient fixation with radiotherapy equipment. We also examined processes in each phase of the emergency response: detecting an emergency, calling the medical emergency team (MET), MET transportation to the site and on-site response and patient transportation to the emergency department. The protocol was modified, and equipment was updated. Onsite simulations were held with and without explanation of the protocol and training scenario in advance. The time for the MET to arrive at the site during a 2017 simulation prior to the present project was $7 \mathrm{~min}$, whereas the time to arrive after the first simulation session was shortened to $5 \mathrm{~min}$ and was then shortened further to $4 \mathrm{~min}$ in the second session, despite no prior explanation of the situation. A multidisciplinary project for emergency response with on-site simulations was conducted at an isolated radiation facility. A carefully planned emergency response is important not only in heavy ion therapy facilities but also in other departments and facilities that do not have easy access to hospital emergency departments.

\section{ABSTRACT}

Gunma University Hospital established the Gunma University Heavy Ion Medical Center (GHMC) in 2010 and has been operating it since then. To date, GHMC has treated more than 4000 patients with cancer. Recently, a patient with no serious underlying medical condition had an unexpected cardiopulmonary arrest (CPA) in a treatment room. Even though this patient was successfully resuscitated, this was the first case of CPA occurring in the GHMC since it was established.

Poor access to the emergency department and immobilisation of patients with a fixation device within the special environment of a radiotherapy facility make it necessary to reconfirm the emergency response. We here report on our quality improvement project, the modification of the protocol, the development of equipment to meet the revised protocol and the dissemination of the system through on-site simulations.

\section{BACKGROUND}

For successful care of sudden life-threatening illnesses, both early detection and a quick response are necessary. Delays in detection or in response times are associated with increased hospital mortality. ${ }^{1}$ Many hospitals have introduced in-house emergency systems and have established a medical emergency team (MET) response for situations that require emergency life support. ${ }^{2} 3$ Protocols for the initial response to an emergency are standardised, and a system for calling for support from the hospital emergency department is established as well as a system for collaborating with support staff and transporting the patient to the emergency department. An effective rapid response system (RRS) or MET can improve survival rates by enabling a rapid response to unexpected cardiac arrest in the hospital. ${ }^{2}{ }^{4-7}$ However, there has been little work on RRSs in hospital areas that have poor access to an emergency department. ${ }^{8}$ In fact, some locations in larger hospitals have poor access to hospital emergency departments, which can result in longer arrival times for emergency staff and lower life-saving rates.

In addition, some facilities have difficulties in implementing an emergency response, 
because, for instance, they lack procedures for early detection or need a special process to initiate cardiopulmonary resuscitation (CPR). Delays in RRS activation are common and influenced by sociocultural issues. ${ }^{9}$ One example is the radiotherapy department. The emergency response to sudden patient emergencies in the radiotherapy department, and its unique problems, have not been previously reported. ${ }^{10-12}$ In a typical radiotherapy treatment room, a special couch and patient fixtures for immobilisation are used, and the patient is left alone in the treatment room during irradiation. Furthermore, many staff, such as medical physicists and accelerator technicians, have little direct contact with the patient and often lack basic life support (BLS) skills. For this reason, it is necessary to prepare for the emergency response in the radiotherapy department in advance.

\section{MEASUREMENT}

While the ultimate goal is to improve patient outcomes, it is not feasible to measure the effectiveness of emergency response procedures using patient outcomes, as sudden CPA in GHMC is a very rare occurrence. In addition, because the nature of the patient's procedure and the timing of transport vary depending on the situation, we measured the time to the MET call and the time to the MET arrival as performance indicators at each simulation session.

As an external benchmark, the response time, which is the time between the call and the arrival of the MET team at the actual site, was calculated based on our hospital's records from 2018 to 2020 and is shown in figure 1 . In almost $95 \%$ of cases, the MET team arrived anywhere in the hospital within 5 min.

\section{DESIGN}

\section{Past efforts and initiatives}

The initial goal of our project was to review previous emergency response efforts at GHMC. SinceGHMC became operational in March 2010, various emergency

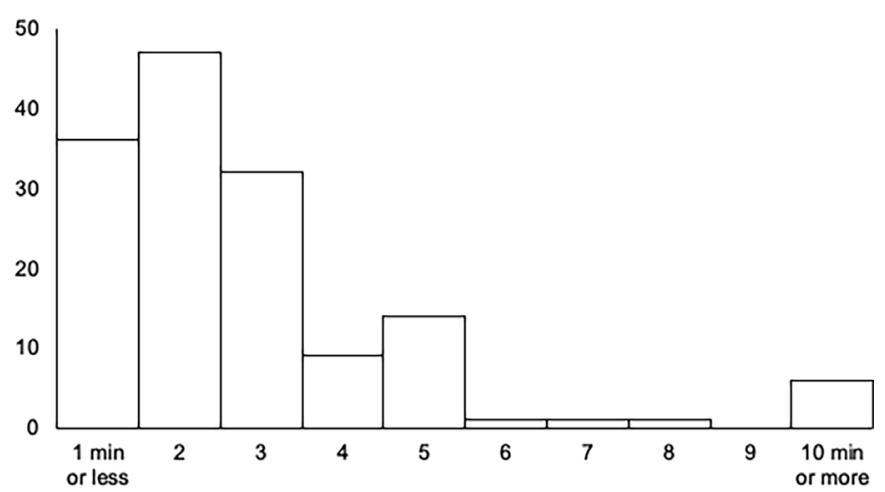

Figure 1 The response time at the actual site. The response time, which is the time between the call and the arrival of the MET team at the actual site, was calculated based on our hospital's records from 2018 to 2020. MET, medical emergency team. response equipment have been installed, as described below, and training has been conducted on an ongoing basis. GHMC-specific training was conducted in addition to hospitalwide standard training. The GHMC-specific training to date has focused mainly on anaphylactic shock following the administration of contrast agent, such as during CT scans. This is because the use of drugs is less common in heavy ion therapy facilities, and although there are many elderly patients and patients with underlying medical conditions, there are few invasive medical procedures.

An automated external defibrillator (AED) was installed in 2010, and, in August 2010, a BLS training exercise was held with 47 participants. After lectures and practice in the use of BLS and AEDs, a role-play situation was conducted, where a patient coming to the facility lost consciousness. The training was conducted in January 2015 with 40 participants. The BLS training was conducted using the hospital's skills lab centre and was followed by a simulation of the initial response and transport to the emergency department, using as a scenario the shock caused by an adverse reaction to a contrast agent during a CT scan.

\section{Creation of the core group}

This quality improvement project was conducted with the collaboration of staff from the GHMC, the hospital emergency department and the Medical Quality and Safety Management Centre. This collaborative group included GHMC staff such as radiation oncologists, nurses, radiologists, physicists and receptionists; MET staff such as emergency department physicians, intensive care unit (ICU) physicians and nurses; and physicians and nurses in the Department of Health Care Quality and Safety Management.

\section{The process for considering improvement measures}

Face-to-face onsite meetings as well as online meetings were held by the core group. First, previous efforts at the GHMC and its unique characteristics as a particle therapy facility were reviewed, and problems in the emergency response were identified. Second, the problems were summarised for each phase of the emergency response, and improvement measures were discussed. Third, the facility's hardware and equipment were renovated. Fourth, the response protocol was revised to adapt it to the renovated facilities.

\section{Protocol modification}

The protocol was revised to clarify the emergency request process initiated by use of the emergency button, with organisation of the measures for each process (figure 2). After the detection of an emergency situation, the emergency button is used to gather the first responders, ensure rapid activation of the MET call from the facility's reception desk and coordinate the MET and patient transport.

On-site simulation

Simulation training sessions were conducted to evaluate the effectiveness of the protocols. In the first session, the 


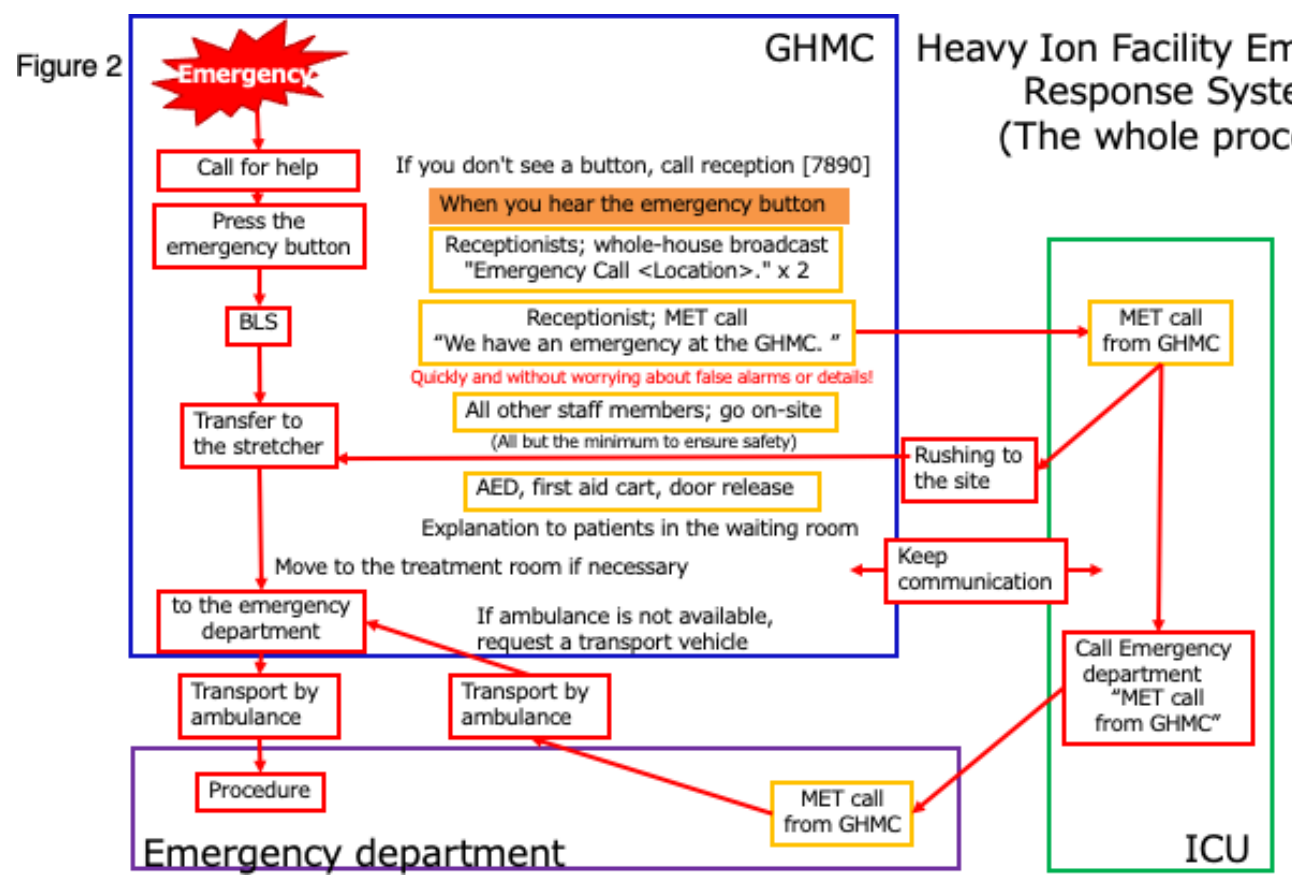

Figure 2 Heavy ion facility emergency response protocol. Illustration of the initial response at a heavy ion irradiation facility, the actions of the MET, and the response in the emergency department. The text in this figure is a modified translation of the original language. AED, automated external defibrillator; BLS, basic life support; ICU, intensive care unit; MET, medical emergency team; GHMC, Gunma University Heavy Ion Medical Center.

protocol was explained in advance, and the scenario was made known to all participants. In this way, problems with the protocol were examined, and its contents were made known to all staff in the GHMC. In the second session, the scenario and implementation date were not given in advance, to ensure a more realistic situation and evaluate the staff understanding and proficiency.

\section{STRATEGY}

\section{Characteristics and background of the heavy ion radiation} therapy facilities

The following three points were indicated to be particularly relevant to the GHMC.

\section{Distance from the emergency department}

The GHMC building is on the same site as the emergency department but is located about $500 \mathrm{~m}$ away from it, and it takes more than $5 \mathrm{~min}$ to walk the distance (figure 3: view of the premises).

Furthermore, the internal structure of the building is complicated, with the entrance being on the mezzanine floor and the treatment rooms on the basement floor, making it difficult for first-time visitors to recognise which floor they are on and to communicate the location over the phone.

Patients are transported between the buildings in a special-care vehicle. The driver is a part-time non-medical worker, and the vehicle is not designed for emergencies, so providing treatment in the vehicle is difficult. Transferring patients also requires the operation of an electric lift, which is slow and time-consuming. In addition, the roads on the campus are mostly one-way, which complicates the transport route to the emergency department.

\section{Many staff have little direct contact with patients}

There are many staff in the facility who are not normally involved with direct patient care, such as accelerator technicians and physicists. Furthermore, some employees are outsourced, and it is necessary to verify their previous

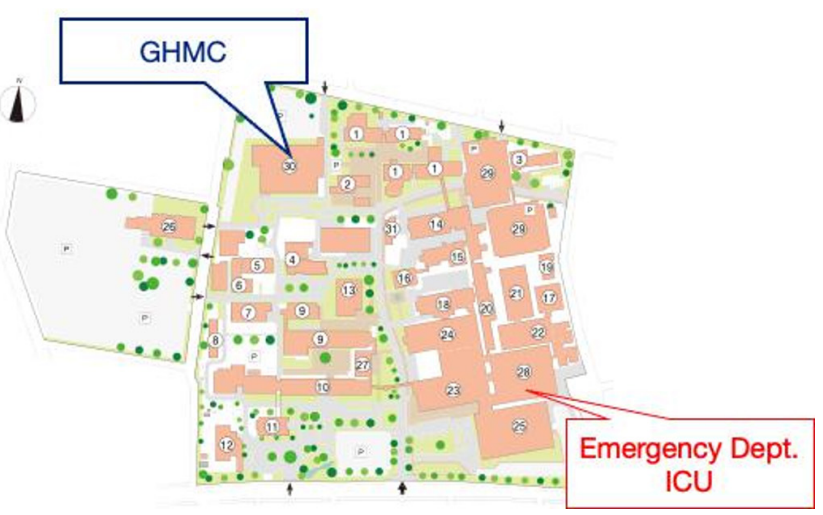

Figure 3 Hospital site plan. The heavy ion facility (GHMC) is separated from the hospital building and is a considerable distance from the hospital's emergency department and the ICU where the MET is based. The campus road is one-way and ambulances in the emergency department need to exit the campus to get to and from the GHMC (Modified from the Gunma University webpage. ${ }^{21} \mathrm{ICU}$, intensive care unit; MET, medical emergency team; GHMC, Gunma University Heavy Ion Medical Center. 
medical emergency training and insure that they have basic life-saving skills.

\section{Treatment room conditions and patient fixation}

As with normal radiation therapy, the patient is immobilised using a fixation device and is left alone in the treatment room during the treatment. The treatment couch is special and rotates, thereby requiring special immobilisation. The patient is also often placed in a prone position, and the immobilisation and prone position make it difficult to monitor the patient's facial expression and breathing condition with video camera monitoring. Furthermore, the patient needs to be removed from the coach to provide resuscitation; however, this process takes time since the patient is fixed by the immobilisation device. The use of electronic equipment in the room is also limited because of the neutron emissions from the treatment beam.

\section{Processes in the emergency response and preparedness}

When we examined the emergency response, we divided the time course from detection of the emergency to transport to the hospital emergency department into five phases.

1. Detecting the emergency.

2. Calling the MET.

3. Transportation of the MET to the site.

4. On-site response.

5. Patient transportation to the emergency department.

For each phase, we identified what needed to be addressed and what action to take.

\section{Detecting the emergency}

During treatment, the patient is left alone. The immobilisation devices used in heavy ion therapy, such as those used for treatment of the head and neck area, typically use a plate shell, which makes it difficult to notice the patient's complexion and facial expressions or any vomiting. This limits the ability to observe the patient through video camera monitoring. Biometric monitoring (SpO2 monitor and ECG monitor) is considered to be useful, but such monitoring is limited because of concerns over equipment failure due to neutron generation in the heavy ion treatment room, and it takes considerable time to setup such monitoring equipment because of the shielding maze. In addition, it was mentioned that it would be necessary to secure additional personnel to check the monitors.

To solve this problem, we introduced antennas for the monitoring devices, a central monitor in the operator's room and a monitoring information-sharing system using tablet terminals. It was also pointed out that the screening of high-risk patients on the basis of comorbidities and treatment methods is also important prior to treatment, to allow detection of changes in the patient's condition.

\section{Calling the MET}

The complexity of the interior structure of the facility is a considerable problem. For example, because of the complexity of the structure, the reach of one's voice is limited. Even if a person shouted for help, it is very possible that no one would hear them. As the number of people in each room is limited, it is difficult to gather support personnel for the initial response. To solve this problem, emergency buttons set to sound the alarm at the staff station in the reception area were placed throughout the building. The existing emergency response protocol was modified to reduce the time taken to contact the MET, and cooperation between the ICU and the emergency department was also confirmed.

\section{Moving the MET}

The MET at our hospital involves a team of ICU doctors and nurses. The route from the ICU to the irradiation facility is long, and the shortest distance needed to be identified in advance. It was also suggested that the internal structure of the building was complex, and guiding was needed after arrival at the facility.

\section{On-site response}

The location and contents of emergency supplies and other necessary items were confirmed. The location of emergency carts and the placement of oxygen masks and tubes were reaffirmed. It was pointed out that it is necessary to regularly confirm the status of each employee's BLS training, including external contract staff.

\section{Transportation to the emergency department}

Patients are transported by our van-type service vehicle between hospital buildings and our facility. For emergency transportation, we decided to use an ambulance, since the van-type vehicle is slow, and treating the patient in the vehicle during transportation is difficult. As the roads on the hospital premises are one way and the routes are limited, other routes using roads outside the premises were discussed. The efficiency of the location for the transfer from the stretcher to the ambulance was also confirmed. The transport and route portions of the protocol were confirmed by specific simulations performed in advance.

\section{On-site simulation}

We performed simulation training using Plan-DoStudy-Act (PDSA). In the first session, the protocol was explained beforehand, and the scenario was made known to all participants. This allowed us to identify problems with the protocol and make the contents of the protocol known. The entire simulation, which assumed a sudden change during treatment, was conducted with the participation of several departments and specialists. The protocol, including the emergency button and notification method, was confirmed to work, and the process ran as envisioned in the planned scenario. Based on the improvements suggested in the postsimulation review of the first session, the second session was conducted in a more realistic situation without prior notification of the scenario or the date of implementation. This session aimed to confirm the staff understanding and protocol 
proficiency. A limited number of informed core staff members were assigned to play the role of the patient and the recorders. The staff member who played the role of the patient wore a bib for identification. The situation was recorded on a preprepared sheet of paper, which was turned over by the staff member as the situation progressed and was complemented verbally.

\section{RESULTS}

Before this study, in January 2017, a simulation of the initial response and transportation to the emergency department was conducted on the assumption that a contrast agent shock occurred during an MRI scan, with the simulation being conducted after a lecture on readiness and contrast agents at GHMC. The simulation showed that the time from discovery to the arrival of support staff was $6 \mathrm{~min}$ and $45 \mathrm{~s}$.

Based on this strategy, we introduced new patient monitoring systems, set the emergency buttons, confirmed MET and patient transportation routes and modified the protocol.

In the first on-site simulation, when the emergency was detected, the emergency button was used to notify staff of the emergency, and 1 min later, an ICU hospital emergency call was made, and staff near to the site responded. Five minutes later, the MET arrived and responded to the location. Nine minutes after the alarm was raised, the patient was transported to the ambulance, and $16 \mathrm{~min}$ after the alarm, the patient arrived at the emergency room (figure $4 \mathrm{~A}$ ).

In the second on-site simulation, a staff member close to the discoverer was notified by the emergency button when an emergency was detected, and within $1 \mathrm{~min}$, an ICU hospital emergency call was made and staff near to the site responded. An ICU team arrived and responded to the location $4 \mathrm{~min}$ after the alarm was raised, the patient was taken to the ambulance at $13 \mathrm{~min}$ after the alarm, and the patient reached the emergency room at 18 min after the alarm (figure $4 \mathrm{~B}$ ).

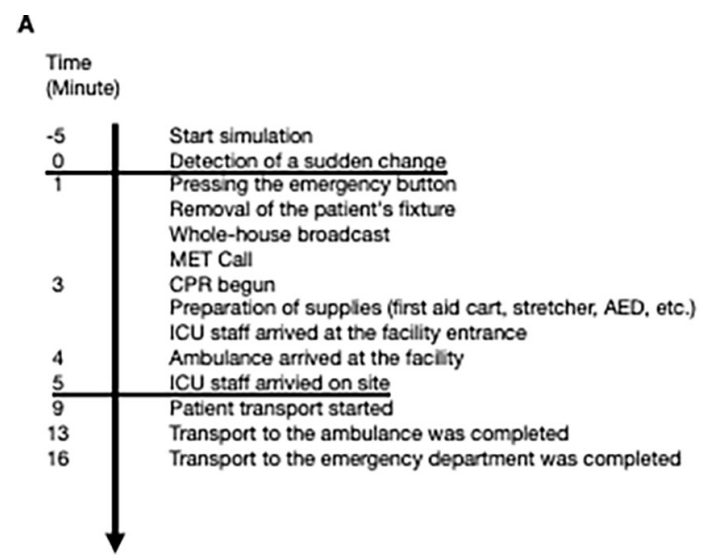

\section{LESSONS AND LIMITATIONS}

A successful RRS requires prompt detection, the sharing of situation awareness among team members, prompt communication to the MET, prompt BLS by the on-site team and effective and efficient resuscitation care by the MET. In this project, we first standardised the equipment and rapid response protocols. The role of all healthcare providers and administrative staff in the radiotherapy unit, as well as the communication flow to the hospital rapid response team, was clearly established. Second, to test the efficacy of the programme, simulated training was repeated, with debriefing sessions to promote the PDSA cycle. The first simulation session was carried out with all participants informed of the protocol and scenario in advance, while the second session was carried out without sharing the scenario, except for a rough schedule. In both simulations, the revised protocols worked, and the equipment established was used. The time for the MET to arrive at the site during the 2017 simulation prior to the present project was $7 \mathrm{~min}$; the time to arrive in the first session was shortened to $5 \mathrm{~min}$, and this was shortened further to $4 \mathrm{~min}$ in the second session, despite the lack of prior explanation of the situation. Although the results are those of simulations and, therefore, make it difficult to make simple comparisons, the reduction in the time to arrival from $7 \mathrm{~min}$ to $4 \mathrm{~min}$ is an outcome of the simulation training, and $4 \mathrm{~min}$ is within the acceptable range when compared with external benchmarks.

An RRS can improve patient safety, and its implementation is recommended in international guidelines. ${ }^{6} 1314$ Gunma University Hospital has an RRS and METs that can reach every unit within $3 \mathrm{~min}$ of the emergency call, except for the GHMC. The absence of any previous cardiac arrest during radiotherapy led to low awareness and preparedness for an RRS among GHMC staff. Facilities with very low incidences of sudden patient deterioration and cardiac arrest should not be excepted from implementing an RRS, standardising the protocol for resuscitation and annual drills to train and monitor the

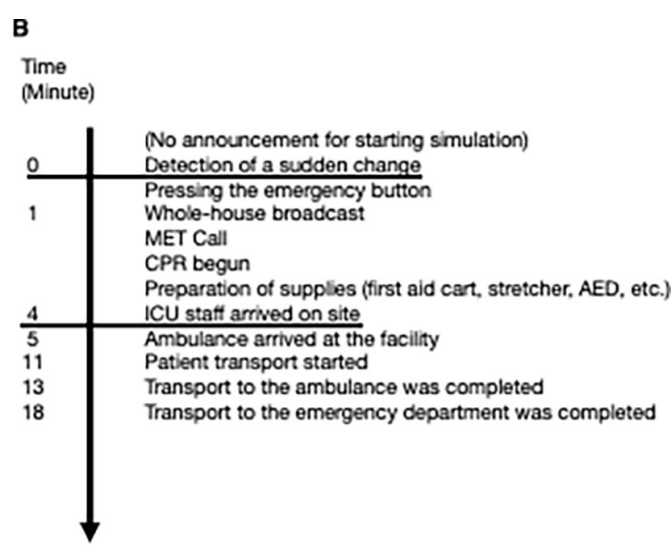

Figure 4 Time course in the simulation. (A) First session, with the protocol and scenario known in advance. (B) Second session, without a prior explanation and only a rough date presented. AED, automated external defibrillator; ICU, intensive care unit; MET, medical emergency team; CPR, cardiopulmonary resuscitation. 
knowledge and skills of the front-line staff. Our experience will be useful for other radiotherapy units as well as for other types of facilities that are located at a distance from the main hospital emergency department.

The planning and implementation of this project required interprofessional collaborations, including between physicians, nurses, radiotherapy technicians, dosimetrists, accelerator technicians, medical physicists and receptionists. Each profession had a different view of the rapid response in the GHMC. One of the valuable lessons learnt through this project is that a major barrier and facilitator for successful resuscitation is communication. ${ }^{15}$ In the postsimulation debriefing, the following problems that should be clarified in advance were pointed out: (1) the initial first responders were medical physicists who did not provide patient care, (2) how staff are notified of the arrival of the MET should be clarified, (3) the shortest route from the ICU to the GHMC should be made clear.

Furthermore, the following problems with the method of conducting the simulation were noted: (1) staff at the centre played the role of the patient, but the personal background of the staff pervaded or confused this role and (2) we presented the scenario on paper and supplemented it verbally, but it was difficult to understand because the patient's situation was not reflected on the patient vital monitor.

Quality improvement and patient safety programmes in radiotherapy units have mostly focused on the accuracy of treatment planning and the avoidance of errors ${ }^{16-19}$; however, there is a paucity of studies focusing on the rapid response to a sudden deterioration in a patient's condition in the treatment room. Although radiotherapy itself is a low-invasive procedure, patients receiving radiotherapy are often old with multiple comorbidities and constitute a vulnerable population. ${ }^{20}$ To assure the quality and safety of radiation therapy, it is critical to develop a RRS that can detect early signs of deterioration in a patient's state and can provide timely and effective resuscitation procedures. Given the rarity of events in the radiation therapy room, the awareness and preparedness for the sudden deterioration in a patient's condition in the radiotherapy unit, including cardiac arrest, could have been considered to be insufficient, at least in our institute. CPA events, although rare, can have a significant impact on patients and also on hospital administrators and staff. Development of an effective RRS for radiotherapy units, especially if they are located at a distance from the hospital emergency or critical care unit, is critical. An on-site simulation-based training programme can detect possible failure modes in the programme, fix the problem and provide confidence among treatment team members. In addition, many staff members rarely have direct contact with patients, so they need special education that takes into account their background knowledge level in medical emergencies and continuous training is important.

The present study has several limitations. First, it is an observational study in a single institution. Although there are only a limited number of facilities that provide heavy ion treatment, the number of facilities is expected to increase in the near future, and our experience will provide useful information for these facilities. Second, although previous reports have shown that delays in the arrival of emergency teams can lead to low lifesaving rates, ${ }^{1}$ the extremely low frequency of sudden cardiac arrest in these facilities prevents an assessment of the actual impact of RRS. Patients in the particle beam facility are undergoing curative treatment for cancer, so their overall health is maintained above a certain level. Actually, as evidenced by the fact that the case that prompted this study was the first CPA case to occur within the GHMC in 10 years, the frequency of such cases is very low, and it is not possible to verify the quantitative improvement in effectiveness using actual CPA cases. Because of this low frequency, our data are limited because it came from simulated training. We plan to conduct annual drill or simulated training to sustain our MET response system. Although we cannot quantitatively prove that our method will improve actual rescue rate, our experience will help other facilities in a similar situation to develop and improve their own RRS and training. Third, it is difficult to measure the overall time lapse in simulations because the length of time required to respond at the initial response location varies depending on the scenario. In this study, we measured the time until the arrival of ICU staff, but we need to be able to examine other indicators. Fourth, staff feedback after the simulation showed an increase in awareness, but it could not be quantitatively evaluated. Ongoing training is required to ensure sustained levels of awareness and preparedness because of the high turnover of staff and the low frequency of serious cases.

\section{CONCLUSION}

A multidisciplinary project to optimise the emergency response with on-site simulations was conducted at an isolated radiation facility. Our experience could be used to develop emergency response systems in other departments and facilities that have difficulty on early emergency detection and poor access to hospital emergency departments. Continuous training is necessary to ensure sustained levels of awareness and preparedness.

Contributors All authors were involved in the design, implementation, analysis and interpretation of the work. HK is involved in the drafting and revising of the manuscript. YK and TO are involved in the revising of the manuscript. HK is state as a guarantor.

Funding The authors have not declared a specific grant for this research from any funding agency in the public, commercial or not-for-profit sectors.

Competing interests None declared.

Patient and public involvement Patients and/or the public were not involved in the design, or conduct, or reporting, or dissemination plans of this research.

Patient consent for publication Not required.

Ethics approval This study does not involve human participants.

Provenance and peer review Not commissioned; externally peer reviewed.

Data availability statement All data relevant to the study are included in the article. 
Open access This is an open access article distributed in accordance with the Creative Commons Attribution Non Commercial (CC BY-NC 4.0) license, which permits others to distribute, remix, adapt, build upon this work non-commercially, and license their derivative works on different terms, provided the original work is properly cited, appropriate credit is given, any changes made indicated, and the use is non-commercial. See: http://creativecommons.org/licenses/by-nc/4.0/.

ORCID iD

Hidemasa Kawamura http://orcid.org/0000-0003-4899-1715

\section{REFERENCES}

1 Calzavacca P, Licari E, Tee A, et al. The impact of Rapid Response System on delayed emergency team activation patient characteristics and outcomes--a follow-up study. Resuscitation 2010;81:31-5.

2 Lyons PG, Edelson DP, Churpek MM. Rapid response systems. Resuscitation 2018;128:191-7.

3 Devita MA, Bellomo R, Hillman K, et al. Findings of the first consensus conference on medical emergency teams. Crit Care Med 2006;34:2463-78.

4 Buist MD, Moore GE, Bernard SA, et al. Effects of a medical emergency team on reduction of incidence of and mortality from unexpected cardiac arrests in hospital: preliminary study. BMJ 2002;324:387.

5 Chan PS, Jain R, Nallmothu BK, et al. Rapid response teams: a systematic review and meta-analysis. Arch Intern Med 2010;170:18-26.

6 Olsen SL, Søreide E, Hillman K, et al. Succeeding with rapid response systems - a never-ending process: A systematic review of how health-care professionals perceive facilitators and barriers within the limbs of the RRS. Resuscitation 2019;144:75-90.

7 Howell MD, Ngo L, Folcarelli P, et al. Sustained effectiveness of a primary-team-based rapid response system. Crit Care Med 2012;40:2562-8.

8 Salman N, Tezel O, Acar YA. Rapid response team performance in Third-Zone, in-hospital outdoor code blue calls. Acibadem Univ Saglik Bilimleri Dergisi 2019;10:260-4.
9 Shearer B, Marshall S, Buist MD, et al. What stops Hospital clinical staff from following protocols? an analysis of the incidence and factors behind the failure of bedside clinical staff to activate the rapid response system in a multi-campus Australian metropolitan healthcare service. BMJ Qual Saf 2012;21:569-75.

10 Ott LK, Pinsky MR, Hoffman LA, et al. Medical emergency team calls in the radiology department: patient characteristics and outcomes. BMJ Qual Saf 2012;21:509.

11 Rudy S, Rooney V, Westley-Hetrick E. Mock codes in a radiology department for systems improvement and staff competency. $J$ Radiology Nurs 2015;34:193-9.

12 Ott LK, Pinsky MR, Hoffman LA, et al. Patients in the radiology department may be at increased risk of developing critical instability. J Radiol Nurs 2015;34:29-34.

13 Kronick SL, Kurz MC, Lin S. Part 4: systems of care and continuous quality improvement. Circulation 2015;132:S397-413.

14 Soar J, Nolan JP, Böttiger BW, et al. European resuscitation Council guidelines for resuscitation 2015: section 3. adult advanced life support. Resuscitation 2015;95:100-47.

15 The Agency for Healthcare Research and Quality. TeamSTEPPS rapid response module. Available: https://www.ahrq.gov/teamstepps/rrs/ instructor_slides/rrsinstructmod.html [Accessed 27 Oct 2020].

16 American Society for Radiation Oncology. Safety is no accident. A framework for quality radiation oncology care, 2019.

17 Adleman J, Gillan C, Caissie A, et al. Development of a quality and safety competency curriculum for radiation oncology residency: an international Delphi study. Int J Radiat Oncol Biol Phys 2017;98:428-37.

18 Yeung A, Greenwalt J. A framework for quality improvement and patient safety education in radiation oncology residency programs. Pract Radiat Oncol 2015;5:423-6.

19 Woodhouse KD, Volz E, Bellerive M, et al. The implementation and assessment of a quality and safety culture education program in a large radiation oncology department. Pract Radiat Oncol 2016;6:e127-34.

20 Sardar M, Shaikh N, Malik SU, et al. Possible predictive factors for in-hospital cardiac arrest in patients with cancer: a retrospective single center study. Cureus 2018;10:e2828.

21 Outline of the graduate school of medicine, graduate school of health sciences, faculty of medicine and universityhospital, Gunma university. Available: https://hospital.med.gunma-u.ac.jp/1_web/wpcontent/uploads/2021/08/gaiyo.pdf [Accessed 2022/1/4]. 